\title{
Spectral Unmixing of Hyperspectral Images in the Presence of Small Targets
}

\author{
Sylvain Ravel, Caroline Fossati, Salah Bourennane
}

Aix Marseille Univ, CNRS, Centrale Marseille, Institut Fresnel, Marseille, France

\begin{abstract}
Generally, the content of the hyperspectral image pixel is a mixture of the reflectance spectra of the different components in the imaged scene. In this paper, we consider a linear mixing model where the pixels are linear combinations of those reflectance spectra, called endmembers, and linear coefficients corresponding to their abundances. An important issue in hyperspectral imagery consists in unmixing those pixels to retrieve the endmembers and their corresponding abundances. We consider the unmixing issue in the presence of small targets, that is, their endmembers are only contained in few pixels of the image. We introduce a thresholding method relying on Non-negative Matrix Factorization to detect pixels containing rare endmembers. We propose two resampling methods based on bootstrap for spectral unmixing of hyperspectral images to retrieve both the dominant and rare endmembers. Our experimental results on both simulated and real world data demonstrate the efficiency of the proposed method to estimate correctly all the endmembers present in hyperspectral images, in particular the rare endmembers.
\end{abstract}

Keywords: Hyperspectral; Unmixing; detection; Rare pixel; Non-negative matrix factorization; Endmember.

\section{Introduction}

Over the last decades, hyperspectral imaging, also known as imaging spectroscopy, has faced a growing interest in multiple fields such as astronomy, agronomy, military, geography ${ }^{[1-4]}$. In hyperspectral images each pixel is acquired by recording images across numerous spectral bands from visible light to infrared, such as the acquired information is an approximation of the reflection spectra of the imaged scene. Given the spatial resolution of the current hyperspectral sensor, a pixel generally contains multiple materials. As each material has a given reflection spectrum, the observed spectrum is a mixture of those materials' spectra called endmembers ${ }^{[5]}$. Hyperspectral unmixing consists in estimating the endmembers present in the dataset and the abundances of those endmembers in each pixel. Unmixing hyperspectral images gives us access to valuable subpixel infor- mation $^{[6]}$. In this paper a linear mixing model ${ }^{[7]}$ is used, in which the endmembers are assumed not to interact with each other. This is a valid approximation on a macroscopic scale ${ }^{[8]}$. The linear mixing model describes the observed pixels as a linear combination of the endmembers, where the linear coefficients are the proportion of each endmember in the pixel.

To deal with the unmixing problem, several methods have been proposed ${ }^{[8,9]}$. The Pure Pixel Index (PPI) ${ }^{[10]}$ method estimates the endmembers using geometrical projection of the dataset. Another geometrical method is the $\mathrm{N}$-finder (N-FINDR) ${ }^{[11]}$ method which determines the minimal volume simplex fitting the observed dataset, the endmembers are then the vertices of this simplex. The Vertex Component Analysis (VCA) ${ }^{[12]}$ method projects data features to find the endmembers. Those three methods depend on the assumption that each endmember is

This is an open-access article distributed under the terms of the Creative Commons Attribution Unported License

(http://creativecommons.org/licenses/by-nc/4.0/), which permits unrestricted use, distribution, and reproduction in any medium, provided the original work is properly cited. 
present and is not mixed in at least one pixel of the dataset. This pure pixel assumption is not always fulfilled. The Independent Component Analysis (ICA) ${ }^{[13]}$ is a statistical approach. This method assumes that the endmembers are independent of one another ${ }^{[14]}$. Generally this hypothesis is not fulfilled in the hyperspectralimages case ${ }^{[14]}$. Another method that uses a statistical approach is the Non-negative Matrix Factorization $(\mathrm{NMF})^{[15]}$ which does not rely on pure pixel assumption. It consists in factorizing the dataset in two matrices. The endmember matrix and the abundance matrix, both subject to non-negativity constraint. The abundance matrix is also subject to an extra sum to one constraint. Numerous methods derivate from NMF have been proposed by using some a priori knowledge ${ }^{[16]}$ or by adding extra constraints such as sparsity constraint ${ }^{[17]}$.

In this paper we focus on hyperspectral images containing possibly two types of endmembers: endmembers present in many, or conversely very few pixels of the entire image. They are called dominant and rare endmembers respectively. While processing hyperspectral images, it is very important to identify a so-called "signal subspace" ${ }^{,[18,19]}$. As shown in ${ }^{[18]}$, it is a difficult task when rare signal components are present in the hyperspectral image ${ }^{[18]}$. Detection of this kind of rare endmembers, which can be seen as anomalies carrying information of interest, remains a challenge for the unmixing methods ${ }^{[20]}$. We propose here a thresholding method based on NMF to detect rare pixels which contains rare endmembers and two unmixing methods based on bootstrap resampling ${ }^{[21,22]}$ and NMF to unmix both dominant and rare endmembers.

The remainder of this paper respects the following organization: Section II describes the linear mixing model and the NMF algorithm. Section III introduces a threshold criterion to detect rare pixels. Section IV presents bootstrap resampling method for hyperspectral unmixing and highlights two ways to unmix both rare and dominant endmembers using bootstrap resampling. Experimental results are available in Section V. Section VI is the conclusion of this paper.

Notations: In the remainder of this paper, the following notations are used: scalars are denoted by italic lowercase roman, like a; vectors by boldface lowercase roman, like a; matrices by boldface uppercase roman, like A.

\section{Materials and methods}

In this section we overview the Non-Negative Matrix Factorization and its limits in presence of rare endmembers or small ground targets. In the following sections we consider a hyperspectral image with $\mathrm{P}$ pixels, $\mathrm{L}$ spectral bands and $\mathrm{N}$ endmembers. We denote the image as the $\mathrm{P} \times \mathrm{L}$ matrix $\mathrm{Y}=\left[\mathrm{y}_{1}, \mathrm{y}_{2}, \ldots, \mathrm{y}_{\mathrm{P}}\right]^{\mathrm{T}}$.

\subsection{Linear mixing model}

In hyperspectral images, pixels are a mixture of different spectral reflectances of materials called endmembers. In the following, we use the linear mixing model to describe those mixtures. Let $\mathrm{y}_{\mathrm{p}} \in \mathbb{R}_{+}^{1 \times \mathrm{L}}$ be a mixed pixel, that is, it contains different materials, of a given hyperspectral dataset with $\mathrm{L}$ spectral bands. Let $\mathrm{N}$ be the number of endmembers in this dataset. The linear mixing model describes this pixel as a linear combination of all the endmembers present in the dataset and the linear coefficients are their corresponding abundances in the pixel. Then the pixel vector $y_{p}$ can be expressed as ${ }^{[7,8]}$ :

$$
\mathrm{y}_{\mathrm{p}}=\sum_{\mathrm{k}=1}^{\mathrm{N}} \mathrm{a}_{\mathrm{pk}} \mathrm{s}_{\mathrm{k}}+\mathrm{n}_{\mathrm{p}}
$$

or

$$
\mathrm{y}_{\mathrm{p}}=\mathrm{a}_{\mathrm{p}} \mathrm{S}+\mathrm{n}_{\mathrm{p}} \quad \mathrm{p}=1, \ldots, \mathrm{P}
$$

where $s_{k}$ is the $k^{\text {th }}$ endmember and $a_{p k}$ is its corresponding abundance in the pixel $\mathrm{p} . \mathrm{n}_{\mathrm{p}}$ is an additive noise vector or model error, and $a_{p}$ is the abundance vector of pixel $\mathrm{p}$.

By rearranging all the $\mathrm{P}$ pixels of the whole hyperspectral image in matrix formation, the mixing model can be expressed as:

$$
\mathrm{Y}=\mathrm{AS}+\mathrm{N}
$$

where each row of $\mathrm{Y} \in \mathbb{R}_{+}^{\mathrm{P} \times \mathrm{L}}$ contains the spectral information of a particular mixed pixel, $\mathrm{S}=\left[\mathrm{s}_{1}, \mathrm{~s}_{2}, \ldots, \mathrm{s}_{\mathrm{P}}\right]^{\mathrm{T}} \in \mathbb{R}_{+}^{\mathrm{N} \times \mathrm{L}}$ is the endmember matrix, $\mathrm{A} \in \mathbb{R}_{+}^{\mathrm{P} \times \mathrm{N}}$ is the abundance matrix whose each row is the abundance vector of the corresponding single mixed pixel. The matrix $\mathrm{N} \in \mathbb{R}_{+}^{\mathrm{P} \times \mathrm{L}}$ is the noise matrix.

To be physically meaningful, the matrices Y, A and $S$ are subject to non-negativity constraint and the abundance vectors are subject to the sum to one constraint expressed as follows ${ }^{[23]}$ :

$$
\mathrm{S} \geq 0, \quad \mathrm{~A} \geq 0, \quad \mathrm{~A} 1_{\mathrm{P}}=1_{\mathrm{P}}
$$

where $1_{P}=[1,1, \ldots, 1]^{T}$ is a vector of $P$ ones.

\subsection{NMF unmixing}

Unmixing hyperspectral images consists in revers- 
ing the mixing model. The NMF is an unmixing method subject to nonnegativity constraint whose aim is to find $\mathrm{A}$ and $\mathrm{S}$ both non-negative, so that ${ }^{[15]}$ :

$$
\mathrm{Y} \approx \widehat{\mathrm{A}} \widehat{\mathrm{S}}
$$

$\widehat{\mathrm{A}}$ and $\widehat{\mathrm{S}}$ are calculated by minimizing the following cost function :

$$
\begin{array}{ll} 
& C(A, S)=\|Y-A S\|^{2} \\
\text { s.t. } & S \geq 0, \quad A \geq 0, \quad A 1_{P}=1_{P}
\end{array}
$$

where $\|.\|^{2}$ is the Frobenius norm.

Solving equation (6) can be done using Alternating Least Squares (ALS) methods ${ }^{[24]}$. They are iterative methods which consist in updating the endmember matrix using a non-negative least square method with a fixed abundance matrix. Then fixing the endmember matrix to update the abundance matrix. Repeating these two steps until convergence. The ALS algorithm is summarized in Algorithm 1.

\section{Algorithm 1 ALS - NMF}

\section{/* Initialization */}

$l \leftarrow 1$

$\mathrm{A}^{(l)} \geq 0$

$\mathrm{S}^{(l)} \geq 0$

/* Iterate until convergence */

while $C\left(\mathrm{~A}^{(l)}, \mathrm{S}^{(l)}\right)$ has not converged do

$$
\begin{aligned}
& \mathrm{A}^{(l+1)} \leftarrow \min _{\mathrm{A} \geq 0} C\left(\mathrm{~A}, \mathrm{~S}^{(l)}\right) \\
& \mathrm{S}^{(l+1)} \leftarrow \min _{\mathrm{S} \geq 0} C\left(\mathrm{~A}^{(l+1)}, \mathrm{S}\right) \\
& l \leftarrow l+1
\end{aligned}
$$

end while

Output: $\mathrm{A}^{(l)}, \mathrm{S}^{(l)}$

\subsection{NMF limitations in presence of rare endmembers}

NMF method relies on the assumption that the total number of endmembers $\mathrm{N}$ is known due to prior knowledge or has been estimated using, for example, signal subspace identification methods ${ }^{[25,26]}$. Due to their rarity, the rare endmembers contribute weakly to the Frobenius norm in equation (6) as compared to the noise contribution. This contribution is weak enough such as decomposing the observed vector pixels onto $N_{d}$ dominant endmembers using NMF algorithm provides an accurate estimation of the dominant endmembers ${ }^{[27]}$. Let $\mathrm{S}_{d}$ denotes the dominant endmembers matrix and $\mathrm{A}_{d}$ be the corresponding abundance matrix. The estima- tion of those two matrices is done by minimizing the following expression:

$$
\begin{gathered}
C\left(A_{d}, S_{d}\right)=\left\|Y-A_{d} S_{d}\right\|^{2} \\
\text { s.t. } \quad \sum_{j=1}^{N_{d}} a_{p j}=1 \quad \forall p
\end{gathered}
$$

where $\mathrm{Y}$ is $P \times L$ matrix in which each row represents a pixel vector.

\section{Proposed method for rare pixels detection}

In this section we assume that an accurate estimation of the dominant endmembers $\widehat{\mathrm{S}}_{d}$ and their abundances $\widehat{\mathrm{A}}_{d}$ are available. Such estimation can be obtained using NMF as described above in section II.C.

\subsection{Reconstruction error from dominant endmembers}

From the dominant endmembers estimation we can reconstruct a hyperspectral data set $\widehat{\mathrm{Y}}_{d}$ such as $\widehat{\mathrm{Y}}_{d}=$ $\widehat{\mathrm{A}}_{d} \widehat{\mathrm{S}}_{d}$. By computing the row-wise, i.e. a pixel-wise, quadratic error between $\mathrm{Y}$ and $\widehat{\mathrm{Y}}_{d}$ we can assess the per pixel reconstruction goodness. The quadratic error $r_{p}$ between a reconstructed pixel and its observed counterpart can be expressed as:

$$
r_{p}=\left\|n_{p}+a_{p} S-\hat{a}_{p d} \widehat{S}_{d}\right\|^{2}
$$

where $\widehat{\mathrm{S}}_{d}$ is the estimated dominant endmember matrix and $\hat{\mathrm{a}}_{p d}$ the estimated corresponding abundance vector. The reconstruction error $r_{p}$ can be expressed as:

$$
r_{p}=\left\|e_{p}+n_{p}\right\|^{2}
$$

where $\mathrm{e}_{p}=\mathrm{a}_{p} \mathrm{~S}-\hat{\mathrm{a}}_{\mathrm{pd}} \widehat{\mathrm{S}}_{d}$ is the estimation error.

When the pixel $p$ contains dominant endmembers only, the estimation error is small, the reconstruction error $\mathrm{e}_{p}$ is in general negligible when compared to the noise contribution, then the reconstruction error can be approximated by $r_{p} \approx\left\|\mathrm{n}_{p}\right\|^{2}$.

However, for the pixels containing rare endmembers, the estimation of dominant endmembers cannot represent those rare endmembers contribution. Then the reconstruction error is generally larger than the sole noise contribution $r_{p} \gg\left\|\mathrm{n}_{p}\right\|^{2}$.

This pixel containing noticeable rare endmembers will have a larger reconstruction error than those containing only dominant endmembers for which the reconstruction error is the sole noise contribution. Thus we can separate the pixels containing rare endmembers from the ones containing only dominant endmembers by defining a threshold value on this reconstruction error. 
In the next section new method to estimate a threshold value is proposed and, following that, a method to identify and localize rare pixels is discussed.

\subsection{Rare pixels identification using proposed thresholding criterion}

In a previous work an empirical threshold to detect rare pixels is used ${ }^{[27]}$. $\mathrm{In}^{[27]}$ we have shown that this threshold value is very sensitive to the level of the abundance values of the rare endmembers, consequently the efficiency of the unmixing is diminished. In order to overcome this drawback, in this paper we propose a method to estimate an optimal threshold value.

If we assume that the noise is a white gaussian vector $\mathrm{n}_{p} \sim \mathcal{N}\left(0, \sigma^{2} \mathrm{I}\right)$, then the norm of the noise vector follows a chi-squared distribution with $L-1$ degrees of freedom:

$$
\left\|\mathrm{n}_{\mathrm{p}}\right\|^{2} \sim \sigma^{2} \chi_{\mathrm{L}-1}^{2}
$$

where $L$ is the number of spectral bands.

In hyperspectral imaging the number of tral bands is large enough to approximate the chi-squared distribution with the normal distribution, such as:

$$
\left\|\mathrm{n}_{\mathrm{p}}\right\|^{2} \sim \mathcal{N}\left(\sigma^{2}, \frac{2 \sigma^{4}}{\mathrm{~L}}\right)
$$

Then the norm of the noise vector depends only on the number of band $L$ and on the noise variance $\sigma^{2}$.

According to the gaussian distribution properties less than $0.3 \%$ of the noise vectors will have a quadratic norm greater than the distribution mean plus three times their variance. So we set the threshold criterion $\tau$ to :

$$
\tau=\sigma^{2}+3 \sqrt{\frac{2 \sigma^{4}}{L}}
$$

This threshold will exclude more than $99.7 \%$ of the dominant pixels.

On the other hand there exist numerous methods to estimate the Signal-to-Noise Ratio (SNR) ${ }^{[26,28]}$ of a hyperspectral image and the SNR formula is given by:

$$
\mathrm{SNR}=10 \log _{10} \frac{\|\mathbf{A S}\|^{2}}{\|\mathbf{N}\|^{2}}=10 \log _{10} \frac{\|\mathbf{A S}\|^{2}}{L P \sigma^{2}}
$$

Thus we can use SNR estimation method to estimate $\sigma^{2}$ :

$$
\hat{\sigma}^{2}=\frac{1}{L P}\|\mathbf{A S}\|^{2} 10^{-\frac{\mathrm{SNR}}{10}}
$$

and set the threshold value using the equation (12). If the reconstruction error $r_{p}$ is superior to the threshold value $\tau$ then the considered pixel must contain rare endmembers which are not unmixed yet. In the following section a method to unmix those rare endmembers is proposed.

\section{Proposed method to unmix rare endmembers}

\subsection{Overview of bootstrap resampling for hyperspectral unmixing}

Bootstrap is a statistical inference method, it was initially used to estimate statistical properties of a population from a sample of this population. Nowadays it generally refers to the resampling method which consists in generating new samples from a single observation sample.

Bootstrap resampling has been used in hyperspectral image classification to solve the class imbalance problems $^{[29]}$. In class imbalance problems some classes are overrepresented in the training set whereas other classes are underrepresented. The bootstrap resampling helps solving this issues by generating new training sets using no other data than the original set. In this case the bootstrap method consists in sampling each class uniformly and with replacement to obtain a new set where each classes are balanced.

When unmixing hyperspectral images in presence of rare endmembers a similar imbalance issue occurs: most of the pixels are dominant pixels and very few of them are rare pixels containing rare endmembers. We propose to use bootstrap resampling to generate new pixels and solve this imbalance.

Bootstrap resampling consists in generating, from the observed data set, a new sample data set with similar property. In this paper the bootstrap method is adapted to hyperspectral unmixing with a linear mixing model to generate a new pixel $\mathrm{y}_{\mathrm{j}}^{*}$. For this we randomly select $q$ pixels from $\mathrm{Y}$ and $q$ coefficients $b_{1, j} \ldots b_{q, j}$ such as $b_{i, j} \geq 0$ and $\sum_{i} b_{i, j}=1$. The new pixel is expressed as follow:

$$
\begin{gathered}
y_{j}^{*}=\sum_{i=1}^{q} b_{i, j} y_{p_{i, j}} \\
\text { s.t. } \sum_{i=1}^{q} b_{i, j}=1 \text { and } b_{i, j} \geq 0
\end{gathered}
$$

where the $p_{i, j}$ are the indexes of the randomly drawn pixels. By introducing the equation (1) of the linear mixing model in the bootstrap equation (15) we obtain the following expression:

$$
\mathrm{y}_{\mathrm{j}}^{*}=\sum_{\mathrm{k}}^{\mathrm{N}}\left(\sum_{\mathrm{i}=1}^{\mathrm{q}} \mathrm{b}_{\mathrm{i}, \mathrm{j}} \mathrm{a}_{\mathrm{i}, \mathrm{j}}\right) \mathrm{s}_{\mathrm{k}}+\sum_{\mathrm{i}=1}^{\mathrm{q}} \mathrm{b}_{\mathrm{i}, \mathrm{j}} \mathrm{n}_{\mathrm{p}_{\mathrm{i}, \mathrm{j}}}
$$




$$
\begin{gathered}
\text { Let } \alpha_{k}=\sum_{i=1}^{q} b_{i, j} a_{p_{i, j} k} \quad \text { and } \quad \tilde{\mathrm{n}}_{j}= \\
\begin{array}{c}
\sum_{i=1}^{q} b_{i, j} n_{p_{i, j}}, \quad \text { then : } \\
\mathrm{y}_{\mathrm{j}}^{*}=\sum_{\mathrm{k}=1}^{\mathrm{N}} \alpha_{\mathrm{j}, \mathrm{k}} \mathrm{s}_{\mathrm{k}}+\tilde{\mathrm{n}}_{\mathrm{j}}
\end{array}
\end{gathered}
$$

As both the $a_{p_{i, j} k}$ and the $b_{i, j}$ have a unitary sum, the sum of the $\alpha_{k}$ is also equal to one. Moreover the term $\tilde{\mathrm{n}}_{j}$ is a weighted sum of noise vectors and still a noise vector with a reduced variance. So the generated pixels follow the same mixing model as the observed pixels, with the same endmembers and a similar additive white gaussian noise of lower variance. Thus by generating $P_{b}$ bootstrap pixels we obtain a new data set $\mathrm{Y}^{*}$ which can be expressed on a matricial form as:

$$
\begin{array}{cc} 
& \mathrm{Y}^{*}=\mathrm{BY} \\
\text { s.t. } & \mathrm{B} \geq 0, \quad \mathrm{~B} 1_{\mathrm{N}}=1_{\mathrm{N}}
\end{array}
$$

where $\mathrm{Y}^{*}$ contains the bootstrapped pixels, $\mathrm{B} \in$ els, B $\in \mathbb{R}^{P_{b} \times P}$ is the randomly drawn bootstrap matrix subject to positivity and sum-to-one constraint and $1_{N}$ is a vector of $N$ ones. It has been shown that the number $\mathrm{q}$ of selected pixels gives better unmixing when $1<\mathrm{q}<$ $\mathrm{N}$ in general case ${ }^{[30]}$. Applying the bootstrap equation (18) to the linear mixing model equation (3) leads to:

$$
\mathrm{Y}^{*}=\mathrm{A}^{*} \mathrm{~S}+\widetilde{\mathrm{N}}
$$

where $\mathrm{S}$ is the endmember matrix which is not modified by bootstrap, $\mathrm{A}^{*}=\mathrm{BA}$ is the resampled abundance matrix and $\widetilde{N}=B N$ is the noise matrix.

\subsection{Estimation of rare endmembers}

In this section we present two ways to estimate rare endmembers from the pixels detected and localized in the HSI using the presented method in the precedent section and the bootstrap resampling introduced in section IV. In the remainder of this section we assume that we have used the rare pixels detection threshold to partition the observed pixels $Y$ into two submatrices: the dominant pixels $\mathrm{Y}_{d}$ from one side and the rare ones $\mathrm{Y}_{r}$ on the other side.

\subsubsection{Bootstrapping both dominant and rare pixels}

The two submatrices $\mathrm{Y}_{d}$ and $\mathrm{Y}_{r}$ can be seen as a classification of the observed pixels into two classes "dominant pixels" and "rare pixels". By definition most of the pixels are dominant ones and very few are rare ones which is the reason why NMF cannot unmix rare endmembers. The key idea is to somehow make the rare pixels being not-rare. For this we use bootstrap resampling ${ }^{[21]}$ method to generate a new image where the "old" rare endmembers are present in abundance.

We generate a new image of $P$ bootstrap pixels. Each bootstrap pixel is generated as explained previously in section IV. To ensure that rare endmembers are not-rare in the bootstrap image we randomly select one pixel in each class, such as:

$$
\mathrm{y}_{\mathrm{j}}^{*}=\alpha_{\mathrm{j}} \mathrm{y}_{\mathrm{d}, \mathrm{j}}+\beta_{\mathrm{j}} \mathrm{y}_{\mathrm{r}, \mathrm{j}}
$$

where $\mathrm{y}_{d, j}$ is chosen randomly from $\mathrm{Y}_{d}$ and $\mathrm{y}_{r, j}$ is randomly selected from $\mathrm{Y}_{r}, \alpha_{j}$ and $\beta_{j}$ are both positive and $\alpha_{j}+\beta_{j}=1$.

As the number of rare pixels in $\mathrm{Y}_{r}$ is very low compared to the number $\mathrm{P}$ of pixels in the image, we can assume that each rare pixel is present in many of the new pixels $\mathrm{Y}^{*}$. Moreover as dominant endmembers are present in a lot of the pixels, by selecting one pixel from $\mathrm{Y}_{d}$ in each bootstrap pixel we ensured that they are present in many of the pixels of $\mathrm{Y}^{*}$. Thus both dominant and rare endmembers are present in the majority of pixels of $\mathrm{Y}^{*}$ and we can use NMF to unmix them by minimizing the cost function:

$$
\mathrm{C}\left(\mathrm{A}^{*}, \mathrm{~S}\right)=\left\|\mathrm{Y}^{*}-\mathrm{A}^{*} \mathrm{~S}\right\|^{2}
$$

By using the NMF method, an estimate of the endmember matrix $\widehat{\mathrm{S}}$ and the bootstrap abundance matrix $\widehat{\mathrm{A}}^{*}$ are estimated.

As the bootstrap abundance matrix $\widehat{\mathrm{A}}^{*}$ is not relevant, the estimation of the abundance matrix corresponding to the original image $\mathrm{Y}$ must be done. For this a non-negative least square method is used to solve:

$$
\widehat{A}=\min _{\Lambda}\|\mathrm{Y}-\mathrm{AS}\|^{2}
$$

And thus we obtain and estimate of the endmembers $\widehat{S}$ and their abundances $\widehat{A}$. The matrix $\widehat{S}$ contains both dominant and rare endmembers. In the remainder of this paper this method which consists in applying NMF to Bootstrapped Dominant and Rare pixels will be called NMF-BDR.

\subsubsection{Bootstrapping rare pixels only}

The NMF-BDR is inspired by what exists in hyperspectral image classification. It relies on an estimate of the dominant endmembers $\widehat{S}_{d}$ to classify pixels between $\mathrm{Y}_{d}$ and $\mathrm{Y}_{r}$ and then estimate all the endmembers of the matrix $\mathrm{S}$.

In this section, we only consider the observed data in $\mathrm{Y}_{r}$. As the matrix $\mathrm{Y}_{r}$ contains very few pixels, most of them are rare pixels or an outlying noise, it may not contain enough pixels to obtain accurate estimation of the rare endmembers with $\mathrm{NMF}^{[27]}$. To deal with this issue we propose to use bootstrap resampling method to 
increase the number of pixels.

Let $\mathrm{Y}^{*}$ be the matrix of the $P_{b}$ bootstrapped pixels upsampled from $\mathrm{Y}_{r}$ :

$$
\mathrm{Y}^{*}=\mathrm{BY}_{\mathrm{r}}
$$

Where $\mathrm{B}$ is the bootstrap $P_{b} \times P_{r}$ matrix as presented in equation (18). The matrix $\mathrm{Y}^{*}$ can be written as:

$$
\mathrm{Y}^{*}=\mathrm{A}^{*} \mathrm{~S}_{\mathrm{r}}+\widetilde{\mathrm{N}}_{\mathrm{r}}
$$

$\mathrm{A}^{*}$ is an artificial abundance matrix corresponding to the bootstraped pixels, but the matrix $\mathrm{S}_{r}$ still contains the expected rare endmembers.

We will apply NMF method to $\mathrm{Y}^{*}$ to minimize the cost function:

$$
\begin{gathered}
C\left(\mathrm{~A}^{*}, \mathrm{~S}_{\mathrm{r}}\right)=\left\|\mathrm{Y}^{*}-\mathrm{A}^{*} \mathrm{~S}_{\mathrm{r}}\right\|^{2} \\
\text { s.t. } \quad \mathrm{S}_{\mathrm{r}} \geq 0, \quad \mathrm{~A}^{*} \geq 0, \quad \mathrm{~A}^{*} 1_{\mathrm{P}_{\mathrm{r}}}=1_{\mathrm{P}_{\mathrm{r}}}
\end{gathered}
$$

We obtain $\widetilde{\mathrm{A}}^{*}$ and $\tilde{\mathrm{S}}_{r}$.

At this point, all the endmembers of the matrix $\widehat{S}$ are estimated. Therefore the non-negative least square method is used such as:

$$
\widehat{A}=\min _{\Lambda}\|Y-A \widehat{S}\|^{2}
$$

The entire proposed method called NMF-BR is summarized in the following in algorithm 2.

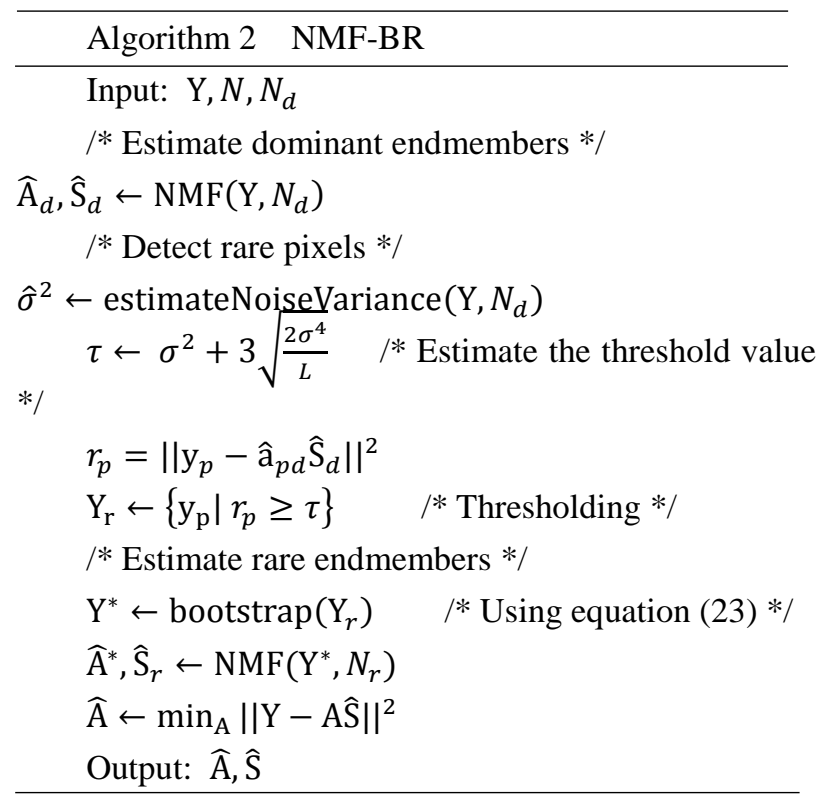

\section{Experiments and results}

\subsection{Performance of the proposed threshold value}

This section compares the empirical threshold ${ }^{[27]}$ and the proposed threshold criterion equation (12). We define the detection rate as the ratio between the number $n_{\text {detected }}$ of rare pixels detected and the number $n_{\text {rare }}$ of rare pixels:

$$
\mathrm{D}=\frac{\mathrm{n}_{\text {detected }}}{\mathrm{n}_{\text {rare }}}
$$

The simulated HSI contains $P=500$ pixels and $L=160$ spectral bands. Pixels are obtained by a linear mixing model with $N_{d}=5$ dominants endmembers and one rare endmembers. $\mathrm{n}_{\text {rare }}=3$ rare mixed pixels are generated by mixing the rare endmember with 2 randomly chosen dominant endmembers according to the model equation (1). A gaussian noise is added to the image to get an expected SNR varying from 16 to $36 \mathrm{db}$. For each value of SNR the detection rate $\mathrm{D}$ is evaluated over multiple simulated data, the averaged results are shown in Figure 1.

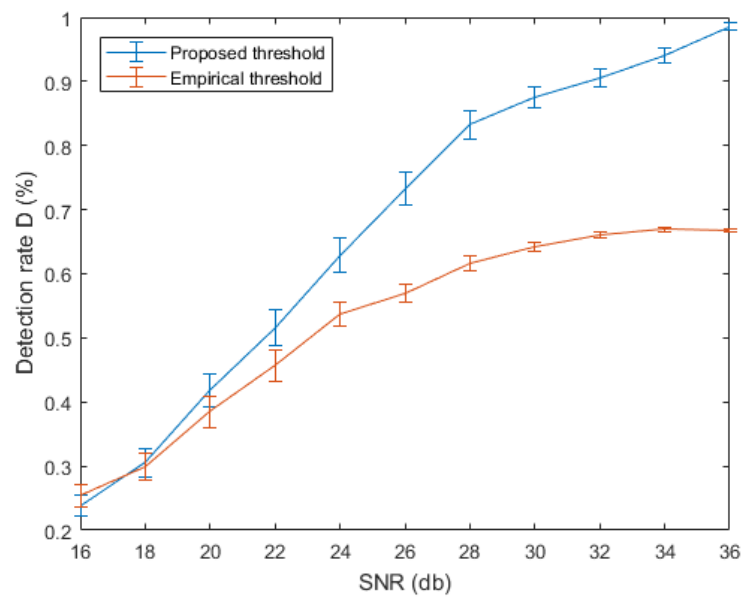

We can see that the proposed criterion has almost always a better detection rate.

Figure 1. Comparison of detection rate D obtained with different methods for different values of the input SNR.

Figure 2 shows the comparison of ROC curves obtained by the proposed threshold and the empirical threshold with SNR $=25 \mathrm{db}$, when adapted for different false alarm rate. It is obvious that the probability of detection values of target detection with the proposed method is improved significantly when compared to the empirical threshold method.

In the considered application missing some rare pixels may cause the loss of important information this why we pretend that the proposed threshold criterion is better suited than the previous one ${ }^{[27]}$. 


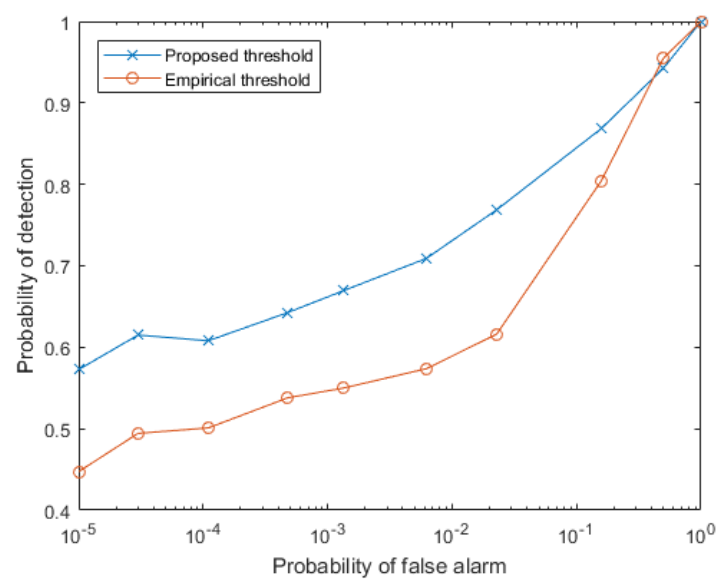

Figure 2. ROC curves obtained by the proposed threshold and the empirical threshold for $\mathrm{SNR}=25 \mathrm{db}$.

\subsection{Evaluation of the proposed unmixing method on simulated data}

This section presents results obtained on simulated hyperspectral images. We generated hyperspectral images of 1600 pixels which contain $N=7$ endmembers of $L=160$ spectral bands from the real-data bank USGS spectral lib ${ }^{[31]}$. The mixing matrix is randomly generated such as two endmembers are present respectively in 16 pixels only $(1 \%)$ and 9 pixels only $(0.6 \%)$ and then can be considered as rare endmembers, we will label those endmembers $\mathrm{s} 1$ and $\mathrm{s} 2$. Therefore there are $N_{d}=5$ dominant endmembers, labelled s3 to s7. A white gaussian noise is added to get a SNR ranging from 24 to $30 \mathrm{db}$. The experiments are run 100 times on different simulated hyperspectral images.

An example of simulated hyperspectral image of size 40x40 pixels is presented in Figure 3. The two rare endmembers are present respectively in four targets with size $2 \times 2$ pixels and one target with size $3 \times 3$ pixels, their abundance map can be shown in Figure 3b.

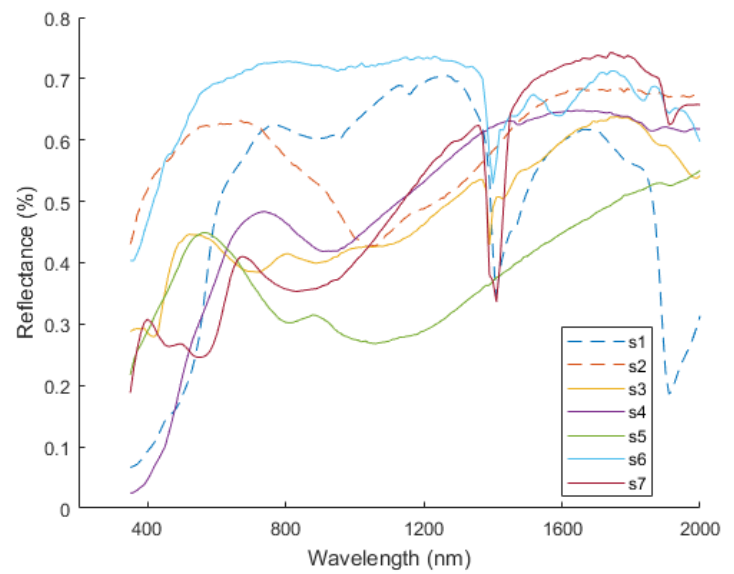

(a) Endmembers used in simulated HSI. Spectra s1 and s2 Remote Sensing (dotted lines) are the rare endmembers. Spectra s3 to s7 are the dominant endmembers.
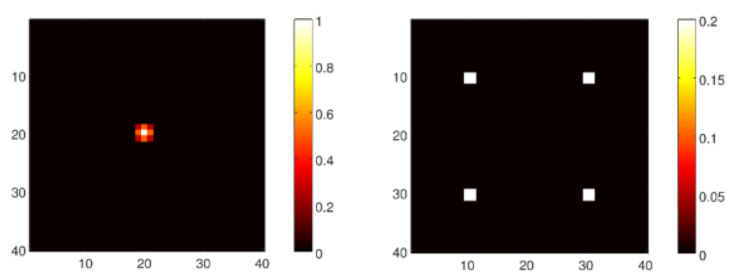

(b) Abundance maps of rare endmembers: ground truth. Above the abundance map of rare endmember $\mathrm{s} 1$. Below the one of rare endmember $\mathrm{s} 2$.

Figure 3. Simulated HSI

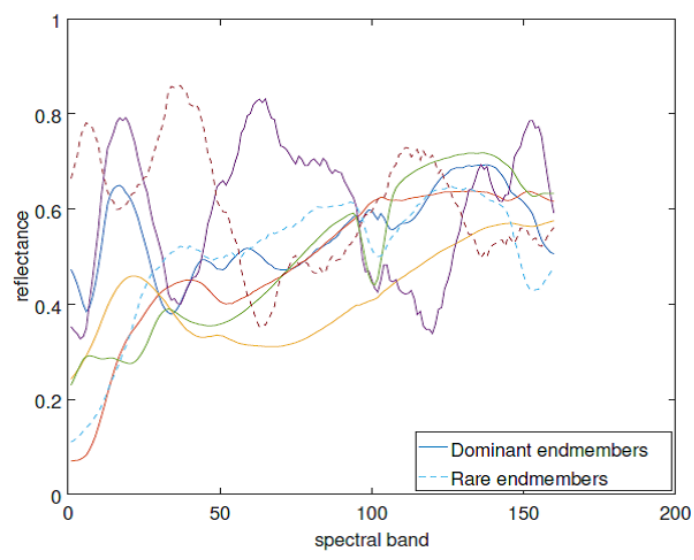

(a) Estimated endmembers with NMF method. Spectra s1 and s2 (dotted lines) are the estimated rare endmembers. Spectra s3 to $s 7$ are the estimated dominant endmembers.
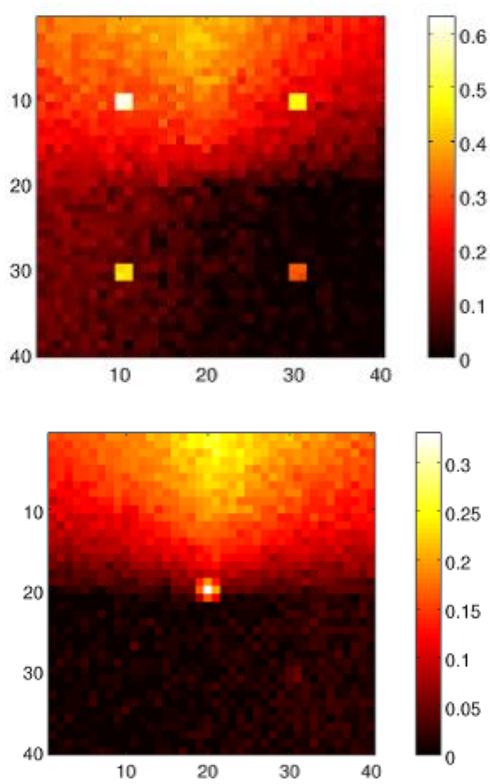

(b) Abundance maps of estimated rare endmembers. Above the abundance map of estimated rare endmember s1. Below the one of rare endmember $\mathrm{s} 2$.

Figure 4. Estimation of endmembers and abundance maps with 
$\mathrm{NMF}$ and $\mathrm{N}=7$.

We use a NMF method to decompose the test set onto 7 endmembers. The estimated endmembers are shown in Figure 4. We also applied the proposed method NMF-BR onto the same test set. The results for the proposed method NMF-BR are presented in Figure 5.

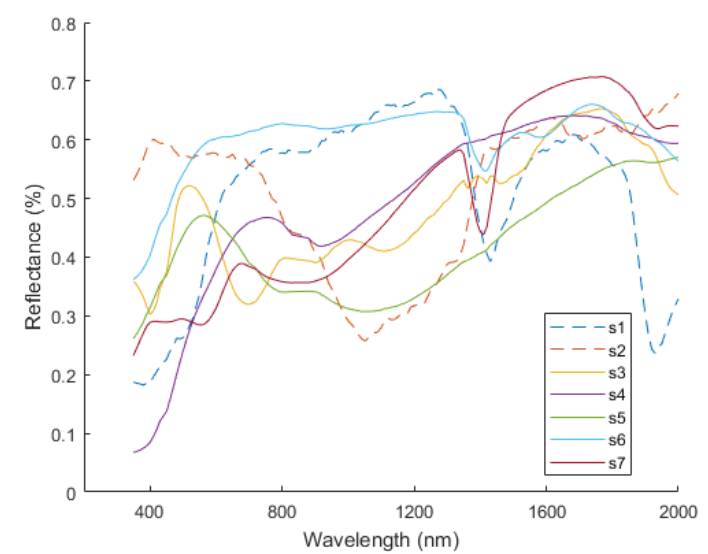

(a) Estimated endmembers with NMF-BR method. Spectra s1 and s2 (dotted lines) are the estimated rare endmembers. Spec-

tra $\mathrm{s} 3$ to $\mathrm{s} 7$ are the estimated dominant endmembers.
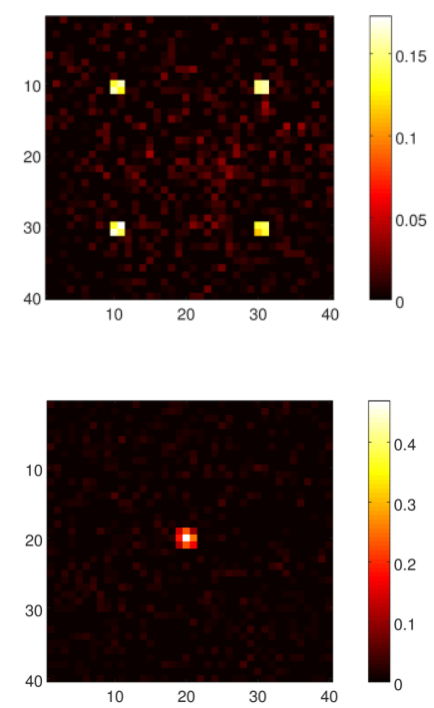

(b) Abundance maps of estimated rare endmembers. Above the abundance map of estimated rare endmember s1. Below the one of rare endmember $\mathrm{s} 2$.

Figure 5. Estimation of endmembers and abundance maps with NMF-BR method

We compare the results obtained with NMF, NMF-BDR and NMF-BR. The endmembers estimation accuracy is assessed with mean spectral angle distance (MSAD):

$$
\operatorname{MSAD}=\frac{1}{\mathrm{~N}} \sum_{\mathrm{k}=1}^{\mathrm{N}} \cos ^{-1}\left(\frac{\mathrm{s}_{\mathrm{k}}^{\mathrm{T}} \hat{\mathrm{s}}_{\mathrm{k}}}{\left\|\mathrm{s}_{\mathrm{k}}|| \mid \hat{\mathrm{s}}_{\mathrm{k}}\right\|}\right)
$$

The MSAD computes the mean angle between the estimated endmembers and the reference endmembers, the lower angle the better. Results are presented in Table 1.

\begin{tabular}{|l|l|l|l|l|}
\hline SNR (db) & $\mathbf{2 4}$ & $\mathbf{2 6}$ & $\mathbf{2 8}$ & $\mathbf{3 0}$ \\
\hline NMF & 0.2656 & 0.2365 & 0.2239 & 0.2033 \\
\hline NMF-BDR & 0.2082 & 0.1961 & 0.1859 & 0.1805 \\
\hline NMF-BR & 0.1958 & 0.1808 & 0.1762 & 0.1645 \\
\hline
\end{tabular}

Table 1. MSAD comparison of NMF, NMF-BDR and NMF-BR methods.

The abundance estimation is assessed using the normalized abundance mean square error (NMSE):

$$
\text { NMSE }=\frac{\|\mathrm{A}-\widehat{\mathrm{A}}\|^{2}}{\|\mathrm{~A}\|^{2}}
$$

The results are given in Table 2 .

\begin{tabular}{|l|l|l|l|l|}
\hline SNR (db) & $\mathbf{2 4}$ & $\mathbf{2 6}$ & $\mathbf{2 8}$ & $\mathbf{3 0}$ \\
\hline NMF & 0.0194 & 0.0195 & 0.0195 & 0.0193 \\
\hline NMF-BDR & 0.0183 & 0.0186 & 0.0184 & 0.0178 \\
\hline NMF-BR & 0.0178 & 0.0178 & 0.0177 & 0.0181 \\
\hline
\end{tabular}

Table 2. NMSE comparison of NMF, NMF-BDR and NMF-BR methods.

Both the rare endmembers and their abundances are accurately estimated by the proposed NMF-BR whereas a classical NMF fails to estimate those rare endmembers. The proposed method NMF-BR is better suited than the NMF and NMF-BDR when small targets or anomalies are present in the hyperspectral image.

\subsection{Experiments with real world data}

The proposed method is now applied to real world data. We use an image from the HYperspectral Digital Imagery Collection Experiment (HYDICE) of size 100x150 pixels and 210 spectral bands ${ }^{[33]}$. The image data were acquired sponsored by the Naval Research Laboratory, by the airborne HYDICE sensor in August 1995 from a flight altitude of $3000 \mathrm{~m}$ with the ground sampling distance approximately $1.5 \mathrm{~m}$. It has $210 \mathrm{spec}-$ tral channels ranging from $400 \mathrm{~nm}$ to $2500 \mathrm{~nm}$ with spectral resolution $10 \mathrm{~nm}$. After removing low SNR bands 1-3, 202-210 and water vapor absorption bands 101-112, $137-153$, there is 169 spectral bands remaining ${ }^{[34]}$. The scene is centred to a large grass field bordered by a road on the right side and a forest in the left side. The field contains 15 small size targets to be identified, organized in a $5 \times 3$ grid where each row is of a different materials 
and each column a different target sizes: $3 \mathrm{mx} 3 \mathrm{~m}, 2 \mathrm{mx} 2 \mathrm{~m}$ and $1 \mathrm{mx} 1 \mathrm{~m}$. Figure 6 shows the scene and Figure 7 corresponds to the targets ground truth.

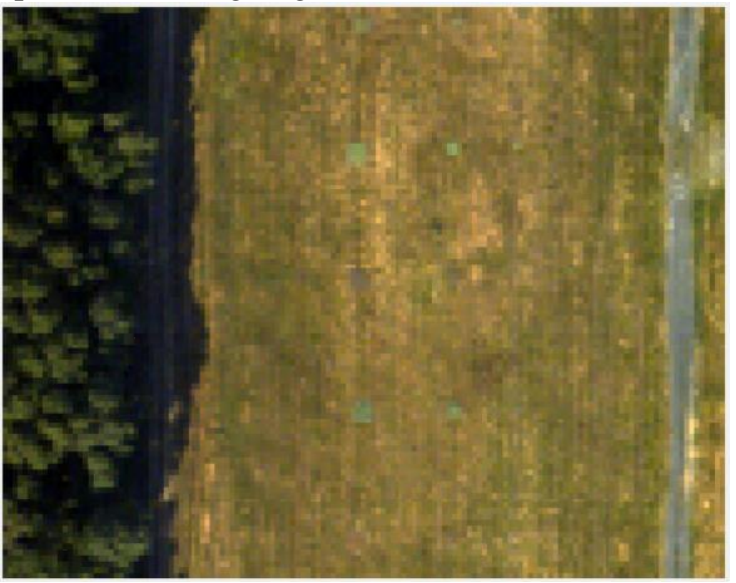

Figure 6. Real World HYDICE HSI.

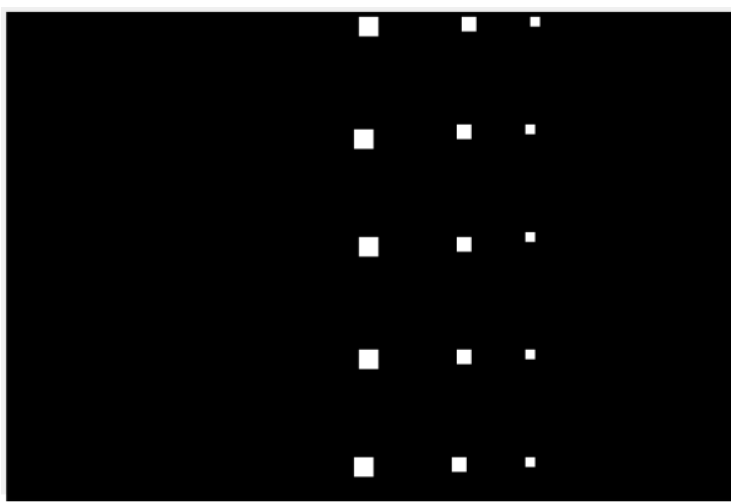

Figure 7.Target locations in the scene.

We use the proposed threshold criterion to detect pixels containing rare endmembers. In the HSI there are 3 dominant endmembers, corresponding respectively to the trees, the grass and the road. Using SNR estimation method $^{[26]}$ the estimated SNR is $30 \mathrm{db}$. The detected pixels are shown in Figure 8. Detected pixels correspond mainly to the targets, the edges of the road and some dirt near the forest.

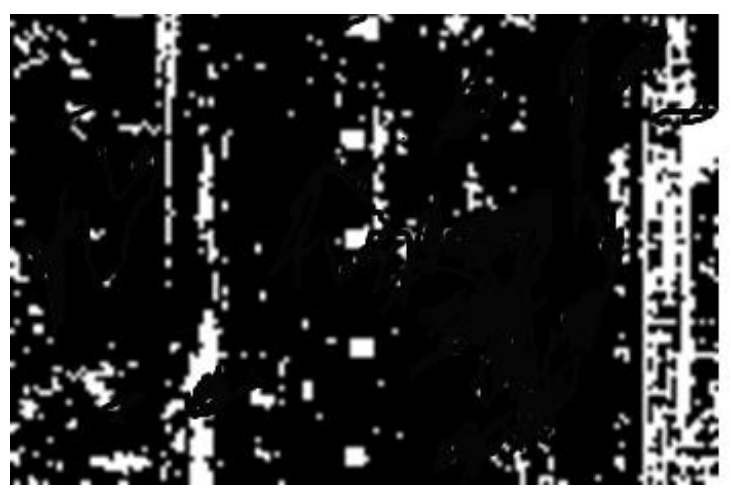

(a)

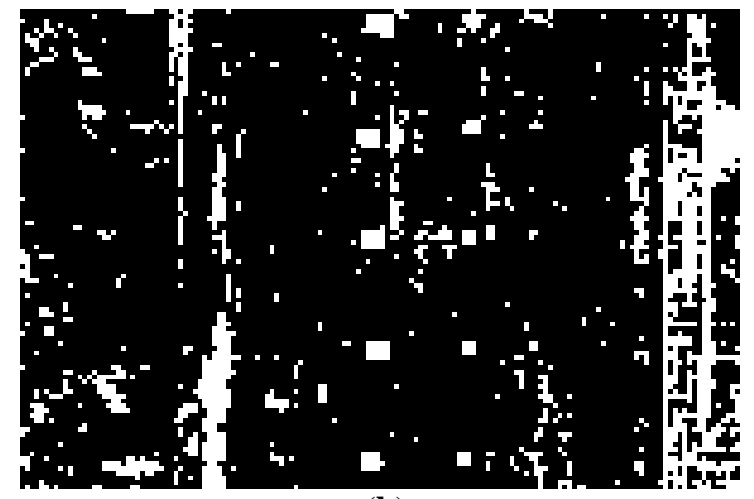

(b)

Figure 8. Detection using : (a) empirical threshold and (b) proposed threshold criterion.

We have applied the presented methods to unmix the hyperspectral images with $\mathrm{N}=8$ endmembers where $N_{d}=3$ endmembers are considered dominant (grass, trees, road) and the other 5 endmembers are the rare ones corresponding to target materials (panels). They are labelled from $\mathrm{t} 1$ for the top panels row, to 5 for the bottom panels row. By averaging the pixels of each target and the pixels of grass, trees and road we estimate their ground truth endmembers. The estimated ground truth endmembers are shown in Figure 9.

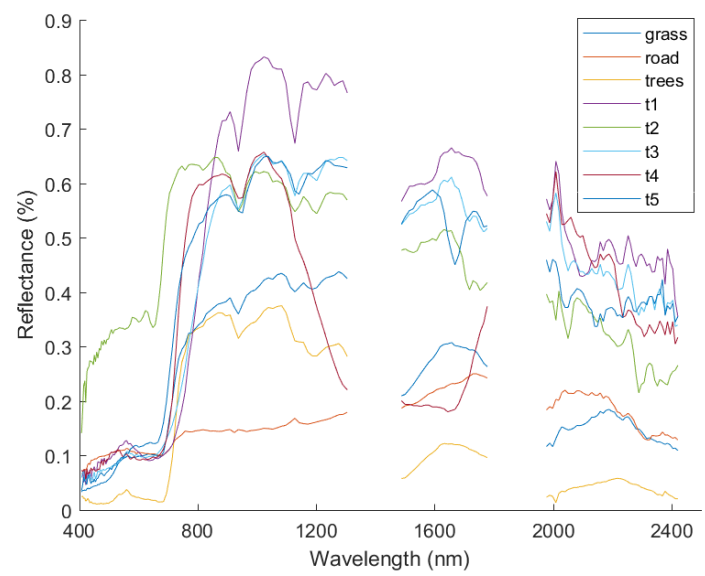

Figure 9. The height ground truth endmembers of the original HSI.

We compute the MSAD between the estimated endmembers and the ground truth endmembers, numerical results are shown in Table III. From these values we can see that the NMF-BR gives better result than the classical NMF and NMF-BDR.

\begin{tabular}{|l|l|l|l|}
\hline & NMF & NMF-BDR & NMF-BR \\
\hline MSAD & 0.5179 & 0.4184 & 0.2715 \\
& & & \\
\hline
\end{tabular}

Table 3. MSAD comparison of NMF, NMF-BDR 
and NMF-BR on HYDICE image.

The abundance map estimated by NMF-BR is presented in Figure 10 where the classes numbered 1-3 represent respectively the grass, the road and the trees and classes 4 to 8 represent the targets. Each pixel has been labelled with the most abundant class. Almost all the targets are detected and localized in the scene. Figure 10 shows also that some pixels are incorrectly labelled near the road (orange) and in the forest (yellow), the main reason is in those areas the endmembers are subject to the variations due to the different shadowing, which is the main reason why the abundances estimated by NMF method are not correct.

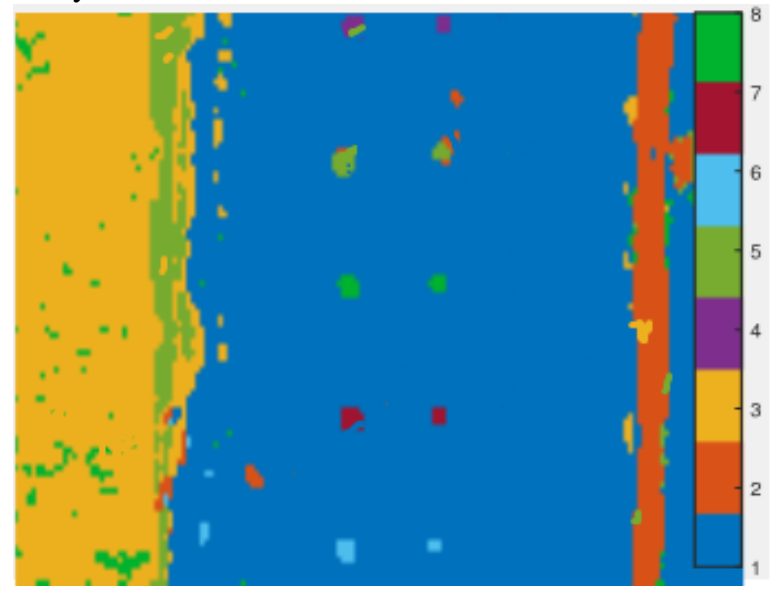

(a)

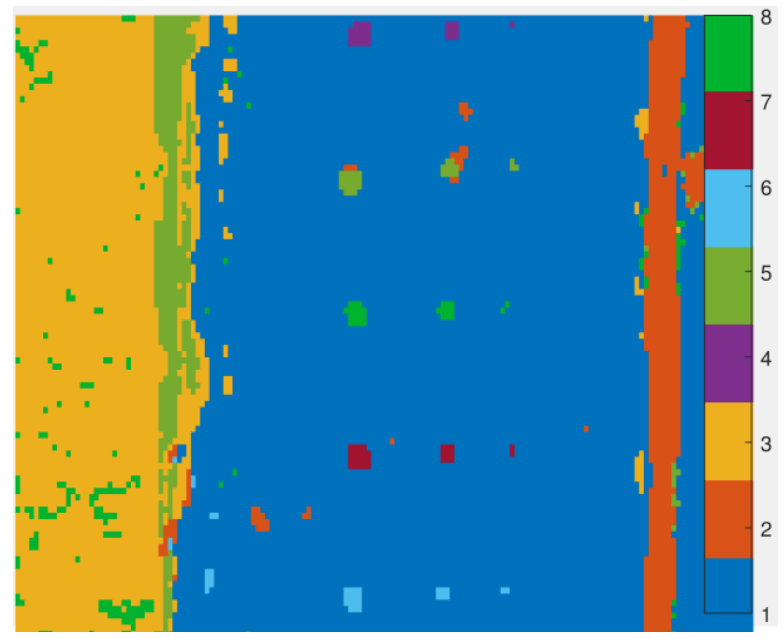

(b)

Figure 10. Abundance map with: (a) NMF method and (b) NMF-BR method. Labels 1, 2 and 3 are respectively the grass, the road and the forest. Labels 4, 5, 6, 7 and 8 are the targets, respectively $\mathrm{t} 1, \mathrm{t} 2, \mathrm{t} 5, \mathrm{t} 4, \mathrm{t} 3$.

\section{Conclusion}

The performances of NMF, NMF-BDR and 10 | Sylvain Ravel et al.
NMF-BR in improving the estimation of endmembers from the linearly mixed pixels of a HSI are discussed in this paper. NMF performs well when the endmembers are present in dominant pixels, it might also miss the rare endmembers. The main explanation is that NMF estimate all the endmembers at once by minimizing the overall reconstruction error.

In this paper we proposed a new thresholding method to detect rare pixels. This method relies on an accurate estimation of the dominant endmembers by NMF and proposes a threshold criterion on reconstruction error which depends on SNR to detect rare pixels whose reconstruction error cannot be explained by the sole noise contribution.

We also proposed two new unmixing methods NMF-BDR and NMF-BR to obtain both dominant and rare endmembers. Those methods are based on NMF and bootstrap resampling. The proposed threshold criterion is used with different bootstrap strategies to increase artificially the abundances of the rare pixels which lead to estimate efficiently the rare endmembers. The NMF-BR method reduces the MSAD up to $50 \%$ compared to the NMF method.

The results on simulated and real world data have shown that the proposed NMF-BR method improves the standard Non-negative Matrix Factorization when the HSI contains rare endmembers, especially, when the targets are with small sizes. The performances of the NMF-BR are highlighted by measures of MSAD and MSE.

\section{Author contributions}

All authors contributed to the conception and design of the methodology.

\section{References}

1. C. C. Borel and S. A. W. Gerstl. Nonlinear spectral mixing models for vegetative and soil surfaces. Remote Sensing of Environment, 47(3), pp. 403416, March 1994.

2. H. J Chauhan and B. K. Mohan. Development of Agricultural Crops Spectral Library and Classification of Crops Using Hyperion Hyperspectral Data. Journal of Remote Sensing Technology, pp. 9-12, May 2013

3. X. Chen, T. A. Warner, D. J. Campagna, Integrating visible, near-infrared and short-wave infrared hyperspectral and mul multispectral thermal imagery for geological mapping at Cuprite, Nevada, Remote Sensing of Environment, 110 (3), pp. 344-356, 2007.

Remote Sensing 
4. S. Asadzadeh, C. R. De Souza Filho, A review on spectral processing methods for geological remote sensing, International Journal of Applied Earth Observation and Geoinformation, 47, pp. 69-90, 2016

5. D. C. Heinz and C.-I-Chang. Fully constrained least squares linear spectral mixture analysis method for material quantification in hyperspectral imagery. IEEE Transactions on Geoscience and Remote Sensing, 39(3), pp. 529-545, March 2001.

6. D. Manolakis, C. Siracusa, and G. Shaw. Hyperspectral subpixel target detection using the linear mixing model. IEEE Transactions on Geoscience and Remote Sensing, 39(7), pp. 1392-1409, July 2001.

7. J. M. Bioucas-Dias, A. Plaza, N. Dobigeon, M. Parente, Q. Du, P. Gader, and J. Chanussot. Hyperspectral unmixing overview: Geometrical, statistical, and sparse regression-based approaches. IEEE Journal of Selected Topics in Applied Earth Observations and Remote Sensing, 5(2), pp. 354-379, April 2012.

8. J. M. Bioucas-Dias, A. Plaza, G. Camps-Valls, P. Scheunders, N. Nasrabadi and J. Chanussot. Hyperspectral remote sensing data analysis and future challenges. IEEE Geoscience and remote sensing magazine, 1 (2), pp. 6-36, June 2013.

9. N. Keshava and J. F. Mustard. Spectral unmixing. IEEE Signal Processing Magazine, 19(1), pp. 44-57, 2002.

10. W. Wang and G. Cai. Endmember extraction by pure pixel index algorithm from hyperspectral image, Proc. SPIE 7157, 2008 International Conference on Optical Instruments and Technology: Advanced Sensor Technologies and tions, 71570E, February 2009. DOI: $10.1117 / 12.811953$

11. M. E. Winter. N-Findr: An algorithm for fast autonomous spectral end-member determination in hyperspectral data. Proc. SPIE 3753, Imaging Spectrometry V, 3753, pp. 266-275, 1999.

12. J.M.P. Nascimento and J.M. Bioucas-Dias. Vertex component analysis: a fast algorithm to unmix hyperspectral data. IEEE Transactions on Geoscience and Remote Sensing, 43(4), pp. 898-910, April 2005.

13. X. Jiang, Y. Jiang, F. Wu, and F. Wu. Quantitative Interpretation of Mineral Hyperspectral Images Based on Principal Component Analysis and Independent Component Analysis Methods. Applied Spectroscopy, 68(4), pp. 502-509, April 2014.

14. J.M.P. Nascimento and J.M. Bioucas-Dias. Does independent component analysis play a role in unmixing hyperspectral data? IEEE Transactions on Geoscience and Remote Sensing, 43(1), pp. 175187, January 2005.

15. M. Arngren, M. N. Schmidt, and J. Larsen. Unmixing of Hyperspectral Images using Bayesian Non-negative Matrix Factorization with Volume Prior. Journal of Signal Processing Systems, 65(3), pp. 479-496, December 2011.
16. W. Tang, Z. Shi, Y. Wu, and C. Zhang. Sparse Unmixing of Hyperspectral Data Using Spectral A Priori Information. IEEE Transactions on Geoscience and Remote Sensing, 53(2), pp. 770-783, February 2015.

17. J. Li, J. M. Bioucas-Dias, A. Plaza, and L. Liu. Robust Collaborative Nonnegative Matrix Factorization for Hyperspectral Unmixing. IEEE Transactions on Geoscience and Remote Sensing, 54(10): pp. 6076-6090, October 2016.

18. N. Acito, M. Diani, and G. Corsini. Hyperspectral Signal Subspace Identification in the Presence of Rare Signal Components. IEEE Transactions on Geoscience and Remote Sensing, 48(4): pp. 1940 1954, April 2010.

19. A. Zidi, J. Marot, S. Bourennane, and K. Spinnler. Bio-Inspired Optimization Algorithms for Automatic Estimation of Multiple Subspace Dimensions in a Tensor-Wavelet Denoising Algorithm. Journal of Remote Sensing Technology, 4, pp. 90-114, December 2016.

20. N. M. Nasrabadi. Hyperspectral target detection : An overview of current and future challenges. IEEE Signal Processing Magazine, 31(1), pp. 34-44, Jan 2014.

21. A. M. Zoubir and D. R. Iskandler. Bootstrap methods and applications. IEEE Signal Processing Magazine, 24(4):pp. 10-19, July 2007.

22. S. Kawaguchi and R. Nishii. Hyperspectral Image Classification by Bootstrap AdaBoost With Random Decision Stumps. IEEE Transactions on Geoscience and Remote Sensing, 45(11), pp. 3845-3851, November 2007.

23. A. Cichocki, R. Zdunek, A.H. Phan, and S.I. Amari. Nonnegative matrix and tensor factorizations: applications to exploratory multi-way data analysis and blind source separation. John Wiley, Chichester, U.K, 2009. OCLC: ocn320432452.

24. R. Zdunek and A. Cichocki. Non-negative matrix factorization with quasi-Newton optimization. In Artificial Intelligence and Soft Computing ICAISC 2006, pp. 870-879. Springer, 2006.

25. O. Kuybeda, D. Malah, and M. Barzohar. Rank Estimation and Redundancy Reduction of High-Dimensional Noisy Signals With Preservation of Rare Vectors. IEEE Transactions on Signal Processing, 55(12), pp. 5579-5592, December 2007.

26. J.M. Bioucas-Dias and J.M.P. Nascimento. Hyperspectral Subspace Identification. IEEE Transactions on Geoscience and Remote Sensing, 46(8), pp. 2435-2445, August 2008.

27. S. Ravel, S. Bourennane, and C. Fossati. Hyperspectral images unmixing with rare signals. In 2016 6th European Workshop on Visual Information Processing (EUVIP), pp. 1-5, October 2016.

28. J. Juan, C. Fossati, and S. Bourennane. Efficient Noise Reduction Method Based on Multilinear Tools for Hyperspectral Imagery. Journal of Remote Sensing Technology, 3(2), pp. 22, 2015.

29. S. Sukhanov, A. Merentitis, C. Debes, J. Hahn, and A. M. Zoubir. Bootstrap-based SVM aggregation 
for class imbalance problems. In 2015 23rd European Signal Processing Conference (EUSIPCO), pp. 165-169, August 2015.

30. C. Fossati, S. Bourennane, and A. Cailly. Unmixing improvement based on bootstrap for hyperspectral imagery. In 2016 6th European Workshop on Visual Information Processing (EUVIP), pp. 1-5, October 2016.

31. J. Kim, Y. He, and H. Park. Algorithms for nonnegative matrix and tensor factorizations: a unified view based on block coordinate descent framework. Journal of Global Optimization, 58(2), pp. 285-319, February 2014.

32. R. N. Clark, G. A Swayze, R. Wise, K. E. Livo, T. Hoefen, R. F. Kokaly, and S. J. Sutley. USGS digital spectral library splib06a. US Geological Survey, Digital Data Series, 231, 2007.

33. R. W. Basedow, D. C. Carmer, and M. E. Anderson, HYDICE system: implementation and performance, In SPIE's 1995 Symposium on OE/Aerospace Sensing and Dual Use Photonics. International Society for Optics and Photonics, 12 June 1995.

34. C. I. Chang, S. S. Chiang, James A. Smith, and Irving W. Ginsberg. Linear spectral random mixture analysis for hyperspectral imagery. IEEE transactions on geoscience and remote sensing, 40(2), pp. 375-392, 2002. 